\title{
MRSA-Associated Lemierre's Syndrome in an Intravenous Drug User
}

\author{
Trilokesh D. Kidambi, $\mathrm{MD}^{7}$, Christopher Lee, $M D, M S c, M P H^{7}$, and R. Jeffrey Kohlwes, MD, MPH $H^{1,2}$ \\ 'Department of Medicine, University of California, San Francisco (UCSF), San Francisco, CA, USA; ${ }^{2}$ Division of General Internal \\ Medicine, Veterans Affair Medical Center, San Francisco, CA, USA.
}

KEY WORDS: MRSA; Lemierre's syndrome; IV drug use; neck abscess. J Gen Intern Med 30(12): 1886-7

DOI: $10.1007 / \mathrm{s} 11606-015-3259-9$

(c) Society of General Internal Medicine 2015

A 24-year-old woman with a history of intravenous drug use presented with 5 days of fevers, rightsided neck pain, and odynophagia. Physical and laboratory examination revealed tender swelling of the right neck and neutrophil-predominant leukocytosis. Computed tomography demonstrated a large right retropharyngeal fluid collection with associated stranding, right internal and external jugular vein thrombi, and multiple peripheral nodular and wedge- shaped pulmonary opacities representing septic emboli (Fig. 1) consistent with Lemierre's syndrome. The patient underwent emergent incision and drainage of the abscess and received broad-spectrum antibiotics. Peripheral blood and abscess cultures grew methicillinresistant Staphylococcus aureus (MRSA). The patient was stable postoperatively and completed 4 weeks of intravenous antibiotics.

Lemierre's syndrome is defined by recent history of painful oropharyngeal infection (anginal illness), evidence of metastatic lesions, and either septic internal jugular thrombophlebitis or isolation of Fusobacterium sp. from blood or sterile sites. ${ }^{1}$ While classically associated with Fusobacterium necrophorum, 14 cases associated with $S$. aureus are reported in the literature, with roughly half occurring in young adults under 32 years of age. ${ }^{2-4}$ In this case, the patient's history of intrave-

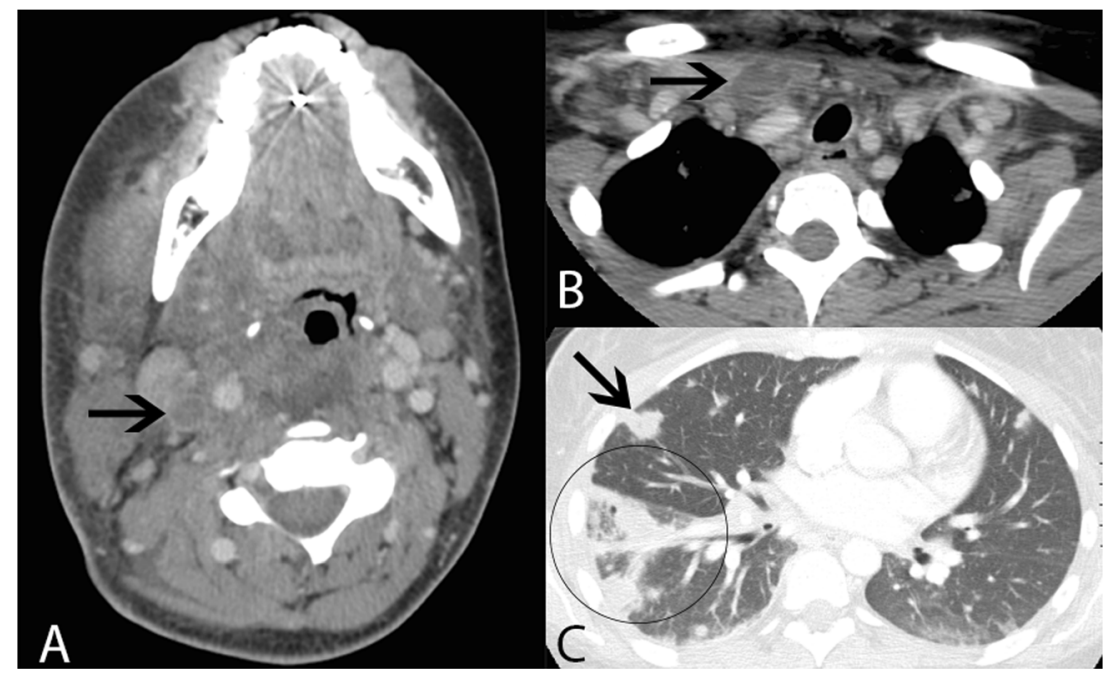

Figure 1 Computed tomography with contrast of the neck (a) and chest (b, c). Panel A shows thrombosis of the right internal jugular vein (arrow). Panel B shows a large abscess (arrow). Panel C shows multiple peripheral nodules (arrow) and a wedge-shaped infarct (circle) consistent with septic pulmonary emboli. 
nous drug use prompted empiric MRSA coverage, , $^{5,6}$ highlighting the importance of risk factor assessment when empirically treating Lemierre's syndrome.

Conflict of Interest: The authors declare that they have no conflicts of interest.

\section{Funding: None}

Corresponding Author: Trilokesh D. Kidambi, MD; Department of MedicineUniversity of California, San Francisco (UCSF), 505

Parnassus Avenue, Room 987, San Francisco, CA 94143, USA (e-mail: Trilokesh.Kidambi@ucsf.edu).

\section{REFERENCES}

1. Riordan T. Human infection with Fusobacterium necrophorum (Necrobacillosis), with a focus on Lemierre's syndrome. Clin Microbiol Rev. 2007;20:622-659.

2. Kizhner V, Samara G, Panesar R, Krespi YP. Methicillin-resistant staphylococcus aureus bacteraemia associated with lemierre's syndrome: Case report and literature review. J Laryngol Otol. 2013;127:721-723.

3. Abhishek A, Sandeep S, Tarun P. Lemierre syndrome from a neck abscess due to methicillin-resistant staphylococcus aureus. Braz J Infect Dis. 2013;17:507-509.

4. Marulasiddappa V, Tejesh CA. Lemierre's syndrome presenting with septic shock. Indian J Crit Care Med. 2013;17:382-384.

5. Mertz D, Viktorin N, Wolbers M, et al. Appropriateness of antibiotic treatment in intravenous drug users, a retrospective analysis. BMC Infect Dis. 2008;8:42-42.

6. Weisse AB, Heller DR, Schimenti RJ, Montgomery RL, Kapila R. The febrile parenteral drug user: a prospective study in 121 patients. Am J Med. 1993;94:274-280. 